\title{
Effets d'une réduction de la teneur en fibres alimentaires sur le transit digestif du lapin. Comparaison et validation de modèles d'ajustement des cinétiques d'excrétion fécale des marqueurs
}

\author{
T Gidenne \\ INRA, centre de recherches de Toulouse, station de recherches cunicoles, BP 27, \\ 31326 Castanet-Tolosan Cedex, France
}

(Reçu le 27 août 1993; accepté le 30 mars 1994)

\begin{abstract}
Résumé - Le transit dans plusieurs compartiments digestifs du lapin a été étudié à partir de 3 aliments à taux décroissants de constituants pariétaux (40,30 et $22 \%$ NDF), distribués à volonté à 4 lapines adultes munies d'une canule à l'iléon. Les temps de séjour moyen (TSM) total et iléo-rectal ont été mesurés simultanément, en suivant l'excrétion fécale de 2 marqueurs ( ${ }^{169} \mathrm{Yb}$ et ${ }^{141} \mathrm{Ce}$ fixés sur la fraction NDF des aliments) distribués en dose unique. Les TSM dans l'ensemble du tube digestif, estimés en appliquant les modèles de Grovum et Williams et celui d'Ellis aux cinétiques fécales de concentration en marqueur, sont similaires à ceux mesurés selon la méthode de référence (formule des moments). Les valeurs de TSM cæcal et stomacal fournies par modélisation sont cohérentes avec celles déduites des mesures de référence. Par rapport au modèle d'Ellis, celui de Grovum et Williams estime plus précisément le TSM dans les compartiments digestifs tubulaires (intestin grêle et côlon distal); en revanche il ajuste moins bien la phase ascendante des cinétiques, surtout dans le cas de régimes pauvres en fibres. La réduction de l'ingestion de fibres $(-50 \%)$ entraîne une hausse du TSM total de $12 \mathrm{~h}(+72 \%)$, provenant principalement d'un doublement du TSM iléo-rectal. Le TSM oro-iléal ( $5 \mathrm{~h}$ en moyenne) n'est globalement pas affecté ; mais le TSM stomacal se raccourcit (de 3 à $1 \mathrm{~h}$ en moyenne), tandis que le transit dans l'intestin grêle est plus long. La baisse de la teneur en fibres ralentit le transit cæcal des particules grossières, sans affecter significativement celui des fines particules.
\end{abstract}

transit / lapin / fibres alimentaires / modélisation

Summary - Effect of a reduction in fibre content on the rate of passage through the digestive tract of the rabbit. Comparison of models for the fecal kinetics of 2 markers. The rate of passage $(R P)$ in several digestive compartments was studied in 4 adult female rabbits cannulated at ileum, and receiving ad libitum 3 diets with decreasing fibre content (40,30 and $22 \%$ NDF). The RP values in the whole digestive tract and between ileum and rectum were simultaneously measured by following the fecal kinetics of 2 markers $\left({ }^{169} \mathrm{Yb}\right.$ and ${ }^{141} \mathrm{Ce}$ adsorbed on the NDF fraction of the diets) given as single doses. The mean retention time (MRT) in the whole tract provided by modelling methods (models: Grovum and Williams or Ellis) were similar to those obtained by reference method (numeric integration). MRT values in the caecum and the stomach were provided by models and were in good agreement with those deduced from reference measurements. Compared with the Ellis model, the 
Grovum and Williams model estimated more precisely the RP in tubular compartments (ie small intestine and distal colon); but the increasing part of the kinetics was better fitted using the Ellis model, particularly for low-fibre diets. The decrease in fibre intake (-50\%) led to a $12 \mathrm{~h}$ increase $(+72 \%)$ of the whole tract MRT, originating mainly from a 2-fold increase of ileo-rectal MRT. The MRT in the stomach decreased from 3 to $1 \mathrm{~h}$, whereas the RP in the small intestine increased. Therefore, the oro-ileal MRT (mean $5 \mathrm{~h}$ ) was not affected. A low dietary fibre induces a longer cecal MRT of large particles, without significant change for fine particles.

rate of passage / rabbit / dietary fibre / model

\section{INTRODUCTION}

L'étude du transit digestif chez le lapin revêt un intérêt au plan nutritionnel et pathologique. Ainsi, un apport élevé de fibres alimentaires accélère le transit mais conduit à une réduction de l'efficacité alimentaire. À I'inverse un apport insuffisant de fibres conduit à un ralentissement du transit, et favorise l'apparition de troubles digestifs mortels (Laplace, 1978). Ces troubles du transit peuvent se manifester dans différents segments digestifs : hypomotilité de l'intestin grêle dans le cas de diarrhée (Fioramonti et al, 1981), mais aussi parésie cæcale (Morisse, 1982 ; Bouyssou, 1987). ll est donc important d'apprécier les variations de temps de séjour des aliments dans chacun des différents segments digestifs. Cette question a été abordée chez le lapin en utilisant la technique des abattages suivie d'une mesure de la concentration en marqueur dans différents segments digestifs (Laplace et al, 1975 ; Furuichi et Takahashi, 1984) ; et seuls Jolivet et al (1975) ont tenté une modélisation des transferts de marqueurs entre compartiments digestifs. Mais, la mesure in vivo du temps de séjour des aliments dans différents segments digestifs a fait l'objet de peu de travaux. Elle nécessite l'implantation de canules pour introduire ou récupérer les marqueurs (Leng et al, 1977 ; Bouyssou et al, 1986 ; Gidenne et al, 1988) ; et il n'est pas envisageable, aujourd'hui, d'implanter plusieurs canules sur un même animal pour étudier simultanément le transit dans plusieurs compartiments digestifs. En outre, la pose de canule iléale n'a été réalisée, jusqu'ici, que chez le lapin adulte. Comme chez le ruminant, l'utilisation de modèles mathématiques paraît alors avantageuse, car ils permettent d'estimer le temps de séjour dans différents compartiments digestifs simplement à partir de l'analyse de la cinétique d'excrétion fécale des marqueurs. Un intérêt supplémentaire des modèles est de fournir une estimation du transit dans les segments digestifs tubulaires, car la mesure directe est imprécise chez le lapin en raison d'une excrétion fécale discontinue liée à la cæcotrophie (Lebas et Laplace, 1974 ; Jilge, 1980). Cependant, la modélisation mathématique de la cinétique de concentration fécale en marqueur nécessite de connaître la signification physiologique des paramètres calculés, en comparant ces estimations à des mesures de référence.

Au plan méthodologique, l'objectif de ce travail a donc été d'évaluer la validité de la modélisation du transit digestif chez le lapin, en comparant des mesures de référence (directes ou par calcul algébrique) obtenues in vivo chez l'animal canulé aux estimations fournies par les 2 principaux modèles mathématiques utilisés chez le ruminant (Grovum et Williams, 1973 ; Ellis et al, 1979). L'incidence de la cæcotrophie sur le transit a également été étudiée à l'aide de paramètres spécifiques.

De plus, ces méthodes ont été appliquées à l'étude des variations du transit dans diférents segments digestifs, lors d'une réduction des apports de fibres alimentaires. 


\section{MATÉRIEL ET MÉTHODES}

\section{Conditions expérimentales}

Trois aliments ont été fabriqués à partir des mêmes ingrédients, selon une formulation simplifiée (tableau I). Ils diffèrent essentiellement par la concentration en constituants pariétaux (CP), sans variation ni de l'origine ni des proportions des différents $C P$. Les aliments ont été distribués successivement, à volonté, à un groupe de 4 lapines adultes pesant $4,0 \pm 0,3 \mathrm{~kg}$, munies de canules à l'iléon terminal (Gidenne et al, 1988).

Les mesures de transit digestif ont été réalisées après $10 \mathrm{j}$ d'accoutumance au régime, en suivant pendant $4 \mathrm{j}$ la cinétique d'excrétion fécale d'une dose de particules (résidu NDF) marquées à l'aide de radiolanthanides (dichlorure de $169 \mathrm{Yb}$ et de ${ }^{141} \mathrm{Ce}$ ). Le résidu NDF de chaque aliment a été marqué séparément avec ${ }^{169} \mathrm{Yb}$ et ${ }^{14} \mathrm{Ce}$ (Gidenne, 1993), afin de suivre simultanément le transit total et iléo-rectal. Une dose $(150 \mathrm{mg})$ de particules marquées avec ${ }^{169} \mathrm{Yb}$ est administrée oralement à $11 \mathrm{~h}$ (TO), afin d'éviter la période de cæcotrophie située en début de matinée. Puis, une dose de ${ }^{141} \mathrm{Ce}$ est introduite dans l'iléon par le biais de la canule à $16 \mathrm{~h}$. Ce délai de $5 \mathrm{~h}$ équivaut au temps moyen de transit dans les parties antérieures du tube digestif (estomac + intestin grêle), et permet de comparer les mesures obtenues avec $\mathrm{Ce}$ et $\mathrm{Yb}$ avec un même écart de temps par rapport à la cæcotrophie. Les fèces ont été récoltées en totalité pendant $96 \mathrm{~h}$, à l'aide d'un collecteur automatique (API, Castanet, France) selon la cinétique de fractionnement suivante: toutes les heures durant les 16 premières heures, puis toutes les $2 \mathrm{~h}$ pendant les $28 \mathrm{~h}$ suivantes, puis toutes les $4 \mathrm{~h}$.

L'ingestion d'aliment est contrôlée quotidiennement et la digestibilité fécale de la matière sèche est calculée à partir de l'excrété sec fécal de la période de mesure du transit (4 i).

La technique d'administration orale des marqueurs, par intubation œsophagienne de particules en suspension dans $5 \mathrm{~cm}^{3}$ d'eau (Gidenne, 1993), a été modifiée afin de minimiser le stress des animaux. La dose de particules marquées est introduite dans un corps de seringue de $1 \mathrm{ml}$, puis humidifiée et comprimée. De ce fait, en quelques secondes, la dose (sous forme d'un granulé) peut être administrée et déglutie sans

Tableau I. Composition centésimale et analyse chimique des aliments expérimentaux.

\begin{tabular}{lrrr}
\hline & $R 1$ & $R 2$ & $R 3$ \\
\hline Ingrédients & & & \\
Luzerne déshydratée & 29,0 & 21,5 & 14,0 \\
Son de blé & 20,0 & 13,2 & 6,5 \\
Paille de blé & 11,0 & 7,0 & 3,0 \\
Blé & 29,0 & 42,6 & 56,2 \\
Tourteau de soja & 9,0 & 13,3 & 17,5 \\
Minéraux et vitamines * & 2,0 & 2,4 & 2,8 \\
Composition chimique (\% sec) & & & \\
Matière sèche & & 89,3 & 89,3 \\
Cendres & 8,2 & 7,4 & 6,8 \\
MAT (N x 6,25) & 17,2 & 18,3 & 19,4 \\
NDF & 39,6 & 30,4 & 10,6 \\
ADF & 20,0 & 15,8 & 2,3 \\
ADL & 4,5 & 3,6 & 9,2 \\
Cellulose brute & 17,3 & 13,3 & \\
\hline
\end{tabular}

* Minéraux (\%) : Cu $(0,35), \mathrm{Zn}(0,77), \mathrm{Mn}(0,33)$; vitamines : A (1,8 $\left.10^{6} \mathrm{Ul} / \mathrm{kg}\right), \mathrm{D} 3\left(210^{5} \mathrm{Ul} / \mathrm{kg}\right), \mathrm{E}(3,3 \mathrm{~g} / \mathrm{kg})$; robénidine : $13,2 \mathrm{~g} / \mathrm{kg}$. 
perte par l'animal, simplement en introduisant la seringue dans la gueule de l'animal.

\section{Calculs des paramètres du transit}

\section{Méthode de référence et mesures directes}

Les temps de séjour moyen de référence (TSMr) des aliments, dans l'ensemble du tube digestif et entre l'iléon et le rectum, ont été calculés algébriquement selon la formule des moments (Faichney, 1975) :

$$
T S M=\Sigma M i . T i / \Sigma M i
$$

où $T i=$ temps écoulé moyen entre l'heure d'administration du marqueur ( $\mathrm{TO}$ ) et la $e$ récolte, et $M i=$ quantité de marqueur excrétée entre $T i-1$ et Ti. Cette méthode de calcul a été prise comme référence pour valider les paramètres obtenus par modélisation, car elle n'impose aucune condition de mesure (état stable, constance de l'excrétion fécale) et ne fait aucune hypothèse sur le fonctionnement dynamique du tube digestif.

Le temps de transit (TT) ou délai d'apparition du marqueur dans les fèces a été défini comme le temps moyen écoulé entre T0 et la première récolte fécale contenant du marqueur. TT correspond au temps minimum nécessaire pour qu'un marqueur traverse les segments digestifs concernés. Cette mesure directe, proposée initialement par Blaxter et al (1956), reflèterait un processus de déplacement linéaire du marqueur sans résidence dans un compartiment de mélange. L'origine physiologique de ce délai est mal identifiée et peut varier selon les auteurs; mais vraisemblablement il correspondrait en grande partie au temps minimum nécessaire pour que le marqueur traverse les parties tubulaires du tube digestif (intestin grêle et côlon distal chez le lapin).

De plus, nous avons calculé la proportion de marqueur excrétée (Ecp) avant la première phase de cæcotrophie qui suit l'administration du marqueur, afin d'évaluer la quantité de marqueur potentiellement recyclable par la cæcotrophie.

\section{Modélisation des cinétiques d'excrétion fécale des marqueurs}

Nous avons appliqué 2 modèles de conception différente aux cinétiques de concentrations fécales des marqueurs distribués oralement : le modèle déterministe de Grovum et Williams (1973) noté $G W$, et le modèle stochastique d'Ellis et al (1979) noté EL. Ces 2 modèles distinguent 2 compartiments de mélange et un compartiment de délai correspondant aux parties tubulaires du tube digestif. Les temps de séjour moyen (TSM) seront respectivement notés : TSM1, TSM2, TTm. Le modèle GW est basé sur une équation de type bi-exponentiel, dont l'inverse des 2 constantes de temps correspond au temps de séjour dans les 2 compartiments de mélange. Le modèle $\mathrm{EL}$ considère que la probabilité de sortie des particules alimentaires est constante dans le premier compartiment de mélange (loi exponentielle, avec une constante de temps K1), mais que dans le second compartiment elle s'accroît avec leur temps de séjour (loi gamma de degré 2 , avec une constante de temps K2) ; TSM1 équivaut alors à $1 / K 1$, et TSM2 à 2/K2.

De plus, dans le but d'analyser l'incidence de la cæccotrophie sur le transit, nous avons défini des paramètres supplémentaires. La pratique de la cæcotrophie est repérable par une absence d'excrétion de fèces dures, au profit de l'excrétion et de l'ingestion immédiate des cæcotrophes (ou fèces molles). Elle a pour effet d'infléchir la décroissance de la concentration (Ct) fécale en marqueur (fig 1), du fait d'un recyclage de particules marquées. Aussi, la phase décroissante de la cinétique a été divisée en 2 parties, avant et après la cæcotrophie qui suit l'administration du marqueur (fig 1). Chaque partie a été ajustée $(\mathrm{CP} 1, \mathrm{CP} 2)$ à une fonction exponentielle $(\mathrm{Ct}=$ Co.exp-kt), dont les inverses des constantes de temps sont respectivement notés TCgp et TCpp. Ces 2 paramètres représentent chacun un temps de séjour moyen dans le second compartiment de mélange (cæcum-côlon proximal). TCgp correspondrait au transit cæcal des particules marquées grossières (>0,3 $\mathrm{mm}$ ) excrétées rapidement dans les fèces dures (Björnhag, 1972 ; Jifge, 1982). Tandis que TCpp correspondrait au transit cæcal des petites particules, refoulées par le côlon proximal en période d'excrétion de fèces dures (Pickard et Stevens, 1972 ; Björnhag, 1981).

\section{Analyses chimiques}

${ }^{169} \mathrm{Yb}$ et ${ }^{141} \mathrm{Ce}$ sont analysés simultanément (spectromètre gamma, Packard 5530) sur la totalité des fèces de chaque récolte, préalablement 


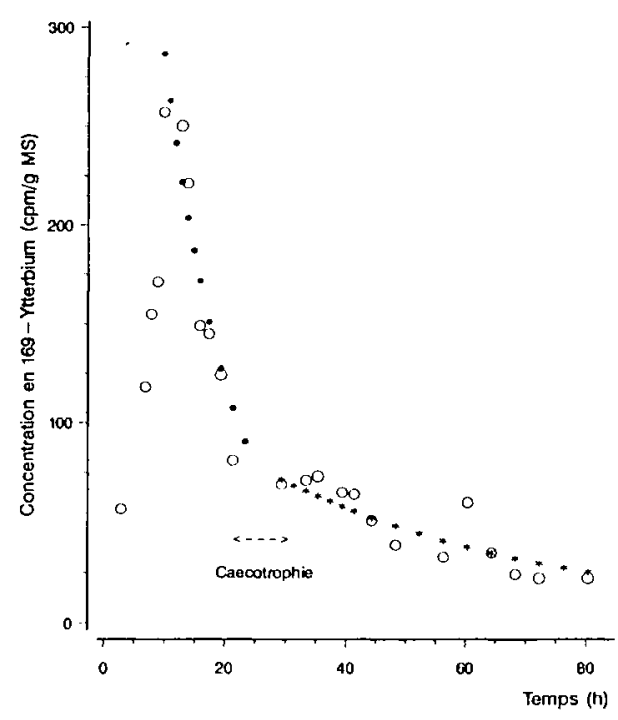

Fig 1. Exemple d'analyse de la cinétique de la concentration fécale en marqueur, en fonction de la cæcotrophie. $\mathrm{O}$ : Valeurs observées. $\bullet$ : Valeurs obtenues selon l'ajustement CP1, avant la première phase de cæcotrophie $(\mathrm{C}=$ Co•exp ${ }^{-k t}, 1 / k=$ TCgp). * Valeurs obtenues selon l'ajustement CP2, après la première phase de cæcotrophie $(C=$ Coeexp-kt, $1 / k=$ TCpp).

séchées, puis pesées. Les analyses suivantes ont été réalisées sur les aliments : matière sèche (MS) par dessiccation durant $24 \mathrm{~h}$ à $103^{\circ} \mathrm{C}$, cendres par calcination durant $5 \mathrm{~h}$ à $550^{\circ} \mathrm{C}$, matières azotées totales (méthode Kjeldahl, $\mathrm{Nx}$ 6,25 ). Les constituants pariétaux (NDF, ADF, et lignine $A D L$ ) ont été analysés séquentiellement selon la méthode de Van Soest et al (1991), après un prétraitement avec une amylase thermostable (Termamyl 120 L, Novo Nordisk, Fontenay, France).

\section{Statistiques}

Chaque cinétique d'excrétion des marqueurs a été analysée individuellement, d'une part par intégration numérique (méthode de référence) pour calculer le TSM total et iléo-rectal, et d'autre part ajustée aux modèles mathématiques (GW et EL) par régression non linéaire, en utilisant la procédure d'itération des moindres carrés de Marquardt (procédure NLIN, SAS 1988 ; Moore et al, 1992). La qualité des ajustements a été contrôlée par la valeur du carré moyen résiduel et lintervalle de confiance des paramètres estimés; de plus un contrôle graphique a été réalisé en comparant la valeur de ces paramètres à leur estimation graphique.

Une analyse de variance à 2 facteurs de classification a été réalisée (procédure GLM, SAS, 1988) afin d'estimer l'effet des régimes sur les paramètres du transit et de tenir compte de la variabilité entre individus. La comparaison des moyennes par régime a été réalisée à l'aide du test de Sheffe. Les écarts de temps de séjour entre les différentes méthodes de calcul ont été comparés 2 à 2 selon le test $t$ de Student.

\section{RÉSULTATS}

\section{Analyse du transit selon la méthode de référence}

La réduction de moitié de la teneur en constituants pariétaux, entre l'aliment $\mathrm{R} 1$ et R3, n'a pas modifié la consommation volontaire des animaux durant les mesures de transit digestif (tableau II) ; en conséquence, l'ingestion de fibres passe de 44,3 à 25,8 $g /$ j. Parallèlement, le temps de séjour moyen (TSM) total des aliments augmente de $12 \mathrm{~h}$ en moyenne $(+72 \%)$, tandis que la digestibilité globale (MS) des aliments augmente significativement $(+22 \%)$. L'allongement du TSM total provient pour une part d'une hausse du temps de transit (TT) total $(+2,8$ h, $P<0,04)$, mais surtout d'un quasi-doublement du TSM iléo-rectal. Par ailleurs, ce dernier est fortement corrélé au TSM total ( $r^{2}$ $=0,981$ ) et à l'apport de NDF $\left(r^{2}=0,49\right)$. Le TSM oro-iléal, obtenu par différence, est en moyenne de $4,6 \mathrm{~h}$, sans variation en fonction des régimes.

La différence entre TT total et iléo-rectal pourrait correspondre à une estimation du 
Tableau II. Effet du taux de fibre sur l'ingestion *, la digestibilité * et le transit digestif total et iléorectal (méthodes de référence).

\begin{tabular}{|c|c|c|c|c|c|c|}
\hline \multirow[b]{3}{*}{ Régimes } & \multirow[b]{3}{*}{ Ingéré $(g / i)$} & \multirow[b]{3}{*}{ CUDa MS (\%) } & \multicolumn{4}{|c|}{ Transit digestif } \\
\hline & & & \multicolumn{2}{|c|}{ Total } & \multicolumn{2}{|c|}{ |léo-rectal } \\
\hline & & & $T S M r$ & $T T$ & $T S M r$ & $T T$ \\
\hline $\mathrm{R} 1$ & 112,0 & $60,6^{b}$ & $16,6^{b}$ & $4,7^{b}$ & $12,8 \mathrm{~b}$ & 3,5 \\
\hline $\mathrm{R} 2$ & 115,5 & 68,9 a & $21,7 \mathrm{ab}$ & $5,8 \mathrm{ab}$ & $16,6 \mathrm{~b}$ & 4,0 \\
\hline R3 & 119,0 & 74,1 a & $28,6^{a}$ & $7,5^{\mathrm{a}}$ & $23,8^{a}$ & 3,6 \\
\hline$S x$ & 3,4 & 1,5 & 1,8 & 0,6 & 1,6 & 0,2 \\
\hline $\mathrm{Pr}>\mathrm{F}$ & 0,17 & 0,006 & 0,010 & 0,039 & 0,007 & 0,28 \\
\hline
\end{tabular}

* Effet déterminé pendant les mesures de transit digestif. TSMr = temps de séjour moyen calculé selon la méthode de référence $(\Sigma m i t i / \Sigma t i) ; T T=$ temps de transit (mesure directe du temps moyen d'apparition du marqueur dans les fèces) ; ${ }^{a, b}$ les moyennes ayant une lettre en commun ne diffèrent pas au seuil $P=0,05 ; \mathrm{Sx}$ : erreur type résiduelle.

TT dans l'intestin grêle (TTi) ; ce dernier passe de $1,1 \mathrm{~h}$ en moyenne à $3,9 \mathrm{~h}$ entre $\mathrm{R} 1$ et R3 $(P<0,07)$. Toutefois, TTi est affecté d'une variabilité assez élevée $(\mathrm{CV} r=60 \%)$, sans doute liée à la relative imprécision de la mesure directe du temps de transit chez le lapin.

\section{Modélisation des cinétiques de concentration fécales des marqueurs}

\section{Précision des ajustements}

L'ajustement des modèles GW et EL aux données expérimentales a été satisfaisant (variance expliquée $=96 \%$ ) et similaire entre modèles (tableau III). Toutefois, les coefficients de variations résiduels des modèles $(\mathrm{CVr})$ sont relativement élevés, en particulier lors de transit rapide avec le régime le plus fibreux (R1). Le plus faible nombre de points de mesure lors d'une excrétion rapide du marqueur est sans doute une des causes de cette baisse de précision. Comparé aux ajustements sur l'ensemble de la cinétique, l'ajustement de la phase décroissante avant la cæcotrophie (CP1) présente une variation résiduelle plus faible et des valeurs plus élevées de variance expliquée. En revanche, l'ajustement CP2 (après la cæcotrophie) apparaît moins précis ( $\mathrm{CVr}=32 \%$ pour R1), probablement en raison des "rebonds" de concentration en marqueur, observés durant cette phase (fig 1).

\section{Comparaison des modèles}

Les TSM dans l'ensemble du tube digestif (TSTm, tableau IV) estimés par le modèle GW ne diffèrent pas de ceux obtenus avec le modèle $E L$. II en est de même pour les TSM dans le second compartiment de mélange (TSM2). Par ailleurs, le coefficient de variation de TSM2 est similaire entre les modèles $\mathrm{GW}$ et $\mathrm{EL}$, et il décroît de R1 à R3 (respectivement $\mathrm{CV}=18$ et $8 \%$ ).

Comparée à TSM2, l'estimation du TSM dans le premier compartiment de mélange (TSM1) est moins précise ( CV moyen = 
Tableau III. Précision des ajustements des cinétiques de concentration en marqueur (doses orales) : variance expliquée (VE) et coefficient de variation résiduel (CVr).

\begin{tabular}{|c|c|c|c|c|c|c|c|c|}
\hline \multirow[b]{3}{*}{ Régimes } & \multicolumn{8}{|c|}{ Ajustements } \\
\hline & \multicolumn{4}{|c|}{ VE (\%) } & \multicolumn{4}{|c|}{$\operatorname{CVr}(\%)$} \\
\hline & $G W$ & $E L$ & $C P 1$ & $C P 2$ & GW & $E L$ & $C P 1$ & $C P 2$ \\
\hline R1 & 96,0 & 96,3 & 98,1 & 96,8 & 26,3 & 25,2 & 18,5 & 32,2 \\
\hline $\mathrm{R} 2$ & 97,5 & 97,5 & 98,4 & 97,4 & 23,4 & 20,8 & $+5,6$ & 22,9 \\
\hline R3 & 97,8 & 97,4 & 98,3 & 97,6 & 18,1 & 20,2 & 13,8 & 19,8 \\
\hline$S x$ & 1,07 & 1,15 & 0,86 & 0,51 & 3,58 & 4,42 & 3,92 & 2,33 \\
\hline $\mathrm{Pr}>\mathrm{F}$ & 0,47 & 0,73 & 0,96 & 0,93 & 0,33 & 0,70 & 0,71 & 0,12 \\
\hline
\end{tabular}

GW : modèle de Grovum et Williams (1973) ; EL : modèle d'Ellis et al (1979) ; CP1 : ajustement de la phase décroissante de la cinétique avant la cæcotrophie qui suit l'administration orale du marqueur (T0) ; CP2 : ajustement de la phase décroissante de la cinétique après la cæecotrophie qui suit TO.

Tableau IV. Influence du modèle d'ajustement GW ou EL sur l'estimation du temps de séjour "TS» (h) dans le premier (TSM1) et le second (TSM2) compartiment de mélange, dans les compartiments tubulaires (TTm), et dans l'ensemble du tube digestif (TSTm).

\begin{tabular}{|c|c|c|c|c|c|c|c|c|}
\hline \multirow[b]{3}{*}{ Régimes } & \multicolumn{8}{|c|}{ Modèles } \\
\hline & \multicolumn{4}{|c|}{ Grovum et Williams } & \multicolumn{4}{|c|}{ Ellis } \\
\hline & TSM1 & TSM2 & $T T m$ & TSTm * & TSM1 & TSM2 & $T T m$ & $T S T m$ * \\
\hline R1 & 2,8 a & $9,7^{b}$ & 3,7 & $16,3^{b}$ & 3,7 & $9,1^{b}$ & 2,9 & $15,6^{b}$ \\
\hline $\mathrm{R} 2$ & $1,6 a b$ & $14,1 \mathrm{ab}$ & 5,3 & 20,9 ab & 2,0 & 14,2 ab & 4,7 & $20,9 a b$ \\
\hline R3 & 1,1 a & $21,5^{b}$ & 5,5 & $28,1^{b}$ & 1,5 & $22,2^{b}$ & 5,4 & 29,1 b \\
\hline$S x$ & 0,3 & 1,9 & 0,8 & 1,9 & 0,6 & 2,0 & 0,8 & 2,1 \\
\hline $\mathrm{Pr}>\mathrm{F}$ & 0,03 & 0,012 & 0,28 & 0,011 & 0,08 & 0,011 & 0,12 & 0,011 \\
\hline
\end{tabular}

* TSTm $=$ TSM1 + TSM2 + TTm ; a,b voir le tableau II.

$30 \%$ ), car elle est basée sur un plus faible nombre d'observations, la phase ascendante de la cinétique étant très rapide (fig 1). Les valeurs de TSM1 obtenues avec le modèle GW sont inférieures $(-0,6 \mathrm{~h} ; P<$ $0,056)$ à celles obtenues avec le modèle EL (tableau V), et sont moins précises pour les régimes à faible taux de fibres $(\mathrm{CV}=22$ et $12 \%$ respectivement pour GW et EL avec le régime $\mathrm{R} 3$ ).

Inversement, le modèle GW donne des valeurs de TS dans le compartiment de délai (TTm : intestin grêle et côlon distal) supérieures $(+0,5 \mathrm{~h} ; P<0,002)$ à celles obte- 
nues avec le modèle EL. De plus, quel que soit le régime, TTm est plus précisément estimé avec GW (CV moyen = 12\%) qu'avec EL (CV moyen $=22 \%$ ).

\section{Comparaison entre modèles et méthode de référence}

Les valeurs de TSM total fournies par les modèles sont sensiblement inférieures $(-0,5 \mathrm{~h}$ en moyenne) à celles de référence (tableau V). Pour le modèle GW, cet écart est constant selon le régime; en revanche avec le modèle EL cet écart est négatif avec le régime $R 1$, puis se réduit (R2) et devient positif pour le régime $R 3$.

La différence entre le TSM et le temps de transit iléo-rectal (tableau II), selon la méthode de référence, est une estimation du TS dans l'ensemble cæcum-côlon proximal. La comparaison de ces valeurs aux estimations obtenues par les modèles pour le second compartiment de mélange (TSM2) indique un écart moyen de 1,1 h en faveur de TSM2. Inversement, les valeurs de TTm données par modélisation sont significati- vement inférieures aux valeurs de temps de transit total (mesure directe), en particulier avec le modèle EL $(-1,7 \mathrm{~h})$; mais ces écarts entre TTm et TT sont variables, pouvant atteindre $5 \mathrm{~h}$.

\section{Analyse du transit en fonction de la cæcotrophie}

L'incidence de la cæcotrophie sur la cinétique d'excrétion fécale des marqueurs (fig 1) se traduit par une décroissance moins rapide des concentrations en marqueurs, voire par une hausse momentanée de cette dernière du fait du recyclage de particules marquées. La quantité de marqueur excrétée avant la phase de cæcotrophie suivante $(E c p)$ ne diffère pas selon que l'administration est orale ou iléale (tableau VI) ; il en est de même pour les valeurs de TCgp et TCpp. L'impact de la cæcotrophie sur les mesures de transit total ou iléo-rectal a donc été similaire, et ceci confirme la validité d'un décalage de $5 \mathrm{~h}$ entre l'heure d'administration orale et iléale du marqueur.

Tableau V. Écarts (h) entre les temps de séjour estimés par les méthodes de référence (Réf) ou par les modèles (GW ou EL) (écart moyen et probabilité de non-nullité entre parenthèse).

\begin{tabular}{lccc}
\hline & & Méthodes \\
& Réf-GW & Réf-EL & \\
& & & GW-EL \\
\hline TSM total & $0,55(0.046)$ & $0,44(0,32)$ & $-0,11(0,73)$ \\
TSM1 & - & - & $-0,57(0,056)$ \\
TSM2 a & $-1,07(0,081)$ & $-1,13(0,14)$ & $-0,06(0,84)$ \\
TTm ${ }^{b}$ & $1,15(0,041)$ & $-1,70(0,006)$ & $0,52(0,002)$ \\
\hline
\end{tabular}

Réf : méthode de référence et mesures directes ; GW et EL : voir tableau III. TSM1, TSM2, TSM total : temps de séjour moyen respectivement, dans le premier, le second compartiment de mélange et dans l'ensemble du tube digestif ; TTm : temps moyen d'apparition du marqueur dans les fèces; a la valeur de "référence" est la différence entre le TSM et le TT mesurés entre iléon et rectum ; b la valeur de “référence» est le temps de transit total (mesure directe, tableau II). 
Tableau VI. Transit digestif en fonction de la cæcotrophie et en fonction de la teneur en fibres des aliments.

\begin{tabular}{|c|c|c|c|c|c|c|}
\hline \multirow[t]{2}{*}{ Régimes } & \multicolumn{3}{|c|}{ Transit total } & \multicolumn{3}{|c|}{ Transit iléo-rectal } \\
\hline & $E_{C P}(\%)$ & $\operatorname{TCgp}(h)$ & $T C p p(h)$ & $E c p(\%)$ & $\operatorname{TCgp}(h)$ & $T C p p(h)$ \\
\hline R1 & $85,3^{b}$ & $6,8^{b}$ & 22,0 & $85,2^{b}$ & 5,3 & 15,1 \\
\hline $\mathrm{R} 2$ & $74,9 a b$ & $9,6 a b$ & 22,5 & $77,0 \mathrm{ab}$ & 6,4 & 20,8 \\
\hline R3 & $60,1^{a}$ & $17,5^{a}$ & 23,8 & 59,6 a & 16,4 & 23,6 \\
\hline Sx & 4,2 & 1,4 & 1,3 & 4,2 & 1,7 & 0,7 \\
\hline $\operatorname{Pr}>F$ & 0,016 & 0,050 & 0,78 & 0,030 & 0,069 & 0,19 \\
\hline
\end{tabular}

Ecp $(\%)=$ proportion de marqueur excrétée avant la cæcotrophie (\% de la quantité totale excrétée) ; TCgp (h) : estimation du temps de séjour moyen cæco-colique des particules grossières, non recyclées par la cæcotrophie TCpp : TCpp (h) : estimation du temps de séjour moyen cæco-colique des fines particules, potentiellement recyclées par la cæcotrophie.

Les valeurs d'Ecp augmentent parallèlement à la hausse du taux de fibres, atteignant plus de $85 \%$ pour R1. Les valeurs de TCgp passent en moyenne de 6 à $17 \mathrm{~h}$ entre $\mathrm{R} 1$ et $\mathrm{R} 3$, tandis que le critère TCpp ne varie pas significativement en fonction des régimes.

\section{DISCUSSION}

\section{Comparaison et validation de modèles}

L'estimation du temps de séjour moyen (TSM) dans l'ensemble du tube digestif est similaire quelle que soit la méthode employée : par intégration numérique des quantités de marqueur excrétées (référence), ou après modélisation des cinétiques de concentration fécale en marqueur. Les écarts $(0,5 \mathrm{~h})$ entre les modèles de la méthode de référence sont faibles et du même ordre de grandeur (mais en sens inverse) que ceux obtenus chez le ruminant (Najar et al, 1990 ; Mambrini, 1990 ; Lallès et al, 1991 ; Bernard, 1992). La modélisation modifie peu la variabilité des mesures de TSM total; quoique la variance résiduelle, calculée lors de la comparaison de régimes, tende à être plus élevée avec le modèle $E L$, comme le constatent aussi Lallès et al (1991).

La mesure directe du temps de transit (TT), en relevant le délai moyen d'apparition du marqueur dans les fèces, est précise si l'excrétion fécale est continue et si les récoltes fécales sont très fréquentes. Or, l'excrétion fécale du lapin en conditions physiologiques est discontinue. Elle suit un rythme nycthéméral dominé par la pratique de la cæcotrophie, qui correspond à une absence d'excrétion de fèces dures pendant plusieurs heures (Lebas et Laplace, 1974 ; Jilge, 1980). De ce fait, la mesure directe de TT (méthode de référence) chez le lapin peut conduire à une surestimation plus ou moins forte du temps de séjour dans les segments digestifs tubulaires. Les valeurs supérieures de TT total par rapport aux estimations fournies par les modèles (TTm) confirment cette hypothèse.

Un intérêt majeur de la modélisation est d'obtenir une estimation du temps de séjour 
des aliments dans les différents compartiments de mélange (estomac, cæcum), simplement à partir de l'analyse des cinétiques de concentration fécale des marqueurs. Mais la validation de ces estimations par rapport à des mesures directes, réalisées chez le lapin porteur d'une canule iléale, demeure délicate étant donné les imprécisions liées à la mesure du TT total et iléorectal. Ainsi, les estimations du TSM dans le second compartiment de mélange (TSM2) fournies par les 2 modèles sont cohérentes avec les estimations pour le cæcum-côlon, obtenues par différence entre le TSM et le TT iléo-rectal (méthode de référence). Cependant, ces dernières sont légèrement inférieures à TSM2, probablement du fait d'une surélévation du TT iléo-rectal.

Dans le cas du TSM stomacal, la validation des estimations données par les modèles est difficile, du fait de la trop grande imprécision de la mesure "directe" qui serait le résultat de plusieurs différences : (TSMtotal-TSMiléorectal) - (TTtotal - TTiléorectal). Cependant, les TSM dans l'estomac estimés par ce calcul sont similaires à ceux obtenus par modélisation pour le premier compartiment de mélange (TSM1) : entre 1,1 et $3,7 \mathrm{~h}$ en moyenne selon les régimes. De plus, signalons qu'une mesure directe du TSM stomacal paraît peu envisageable chez le lapin étant donné la difficulté d'implantation d'une canule duodénale. Jusqu'alors, seules des mesures indirectes après abattage (Gidenne, 1985, 1987) ont été réalisées; et les valeurs obtenues (2 à $3 \mathrm{~h}$ ) sont équivalentes à TSM1. Par ailleurs, il est difficile d'attribuer une supériorité d'un modèle par rapport à l'autre pour estimer le TSM stomacal, car la précision de l'ajustement dépend de la nature du régime. En effet, la phase ascendante des cinétiques, moins rapide avec les régimes fibreux, est mieux décrite par le modèle $E L$, en accord avec Mambrini (1990). Mais les variations résiduelles de TSM1 lors de la comparaison des régimes sont plus fortes avec le modèle $E L(S x=0,6)$, comparé à $G W$ $(\mathrm{Sx}=0,3)$.

\section{Incidence de la cæcotrophie sur les mesures de transit}

La cæcotrophie correspond à un recyclage d'une partie du contenu cæcal (indépendamment de la taille des particules) sous forme de cæcotrophes, conduisant donc à allonger le temps de séjour des digesta recyclés. Ainsi, lorsque l'on ne permet pas au lapin d'ingérer les cæcotrophes, on observe une réduction du temps de séjour des marqueurs (Piekarz, 1963 ; Luick et al, 1992). Mais, la cæcotrophie, en soi, ne modifierait pas le transit intestinal (Jilge, 1974) ou iléorectal (Leng et al, 1977). En accord avec Nielsen (1991), nous constatons que l'allure de la cinétique des marqueurs est modifiée après la première phase de cæcotrophie suivant T0 (fig 1). Il est donc plus précis de réaliser un double ajustement de la phase décroissante de la cinétique avant et après la cæcotrophie, contrairement à un ajustement unique comme le proposent Sakaguchi et al (1992). Le fait que TCgp et TCpp ne diffèrent pas selon que le marqueur est administré oralement ou dans l'iléon, confirme que ces critères correspondent bien à un TSM des particules marquées dans l'ensemble cæcum-côlon proximal.

\section{Effet de la teneur en fibres alimentaires}

De manière générale, le lapin en croissance nourri à volonté réduit son niveau d'ingestion lors d'une réduction de la teneur en fibres dans l'alimentation. Mais, dans nos conditions expérimentales, le niveau d'ingestion des animaux varie peu, probablement du fait qu'il s'agit ici de lapins adultes à l'entretien, dont les besoins nutritionnels sont couverts, y compris avec le régime le plus fibreux. II n'est pas non plus exclu que la 
régulation de l'appétit soit modifiée chez les animaux porteurs d'une canule iléale. L'effet de la teneur en fibres sur le transit digestif est donc, ici, indépendant d'une variation du niveau d'ingestion, sans avoir eu recours à un rationnement.

La réduction des apports de fibres, sans modification de leur nature, a pour principal effet d'allonger le temps de séjour dans l'ensemble cæcum-côlon, en accord avec les résultats de Gidenne et al (1990) obtenus avec des régimes semi-purifiés, alors que, chez le lapin rationné, la teneur en fibres paraît peu modifier le temps de séjour cæco-colique (Gidenne, 1993).

Le transit oro-iléal n'est pas affecté par une baisse du taux de fibres alimentaires. Cependant, les estimations de TSM par modélisation indiquent un ralentissement du transit dans l'intestin grêle, compensé par un transit stomacal plus court. Cet effet des fibres sur la vidange gastrique a été observé antérieurement chez le lapin en croissance, par la technique des abattages (Gidenne, 1987 ; Herrmann, 1989). Ceci confirmerait donc l'existence d'une régulation du transit oro-iléal chez le lapin en fonction de la teneur en fibres alimentaires.

La quantité de fibres ingérées ne semble pas avoir d'effet majeur sur le temps de séjour cæcal des fines particules, ce qui reflète une adaptation de l'activité motrice anti-péristaltique du côlon proximal en fonction de l'apport de fibres. En revanche, le renouvellement cæcal des particules grossières est fortement réduit par une baisse de la teneur en fibres.

\section{CONCLUSION}

Cette première étude portant sur la modélisation de la cinétique d'excrétion fécale des marqueurs chez le lapin a permis de valider les modèles par rapport aux méthodes de référence et aux mesures directes, concernant le temps de séjour dans l'ensemble du tube digestif et dans les principaux compartiments tels que le cæcum et l'estomac. Par rapport au modèle d'Ellis, le modèle déterministe de Grovum et Williams semble fournir des estimations plus précises du temps de séjour dans les compartiments fubulaires ; en revanche, il semble moins bien ajuster la phase ascendante des cinétiques associées à des régimes pauvres en fibres.

L'emploi conjoint des 2 types d'analyses du transit des particules alimentaires a permis de suggérer la présence d'une régulation du transit oro-iléal, en fonction de la teneur en fibres du régime. Ainsi, chez le lapin nourri à volonté, la réduction de l'apport de fibres ne modifie pas le transit oro-iléal ; mais le transit stomacal apparaît plus rapide, et compensé par un transit intestinal plus lent. Cependant, l'effet majeur des fibres est d'accélérer le transit cæcal, en particulier le renouvellement des particules grossières.

\section{REMERCIEMENTS}

Claude Poncet (SRNH, unité Dynamique de la digestion, INRA-Theix) est remercié pour la lecture critique de ce travail, ainsi que Laurence Bernard (SRNH, unité Dynamique de la digestion, INRA-Theix) pour ses conseils liés à l'utilisation des procédures de régression non linéaire du logiciel SAS. André Lapanouse (INRA, station de recherches cunicoles) est remercié pour les soins apportés aux animaux.

\section{RÉFÉRENCES}

Bernard L (1992) Étude de la dynamique des particules et des liquides dans le réticulo-rumen, chez le mouton recevant une ration de foin de dactyle sous forme hachée ou broyée en différentes proportions. Thèse de doctorat, Univ Clermont-Ferrand, nov $1992,180 \mathrm{p}$

Björnhag $G$ (1972) Separation and delay of contents in the rabbit colon. Swed J Agric Res 2, 125-136 
Björnhag G (1981) The retrograde transport of fluid in the proximal colon of rabbits. Swedish J Agric Res 11, $63-69$

Blaxter KL, Graham NMc, Wainman FW (1956) Some observations on the digestibility of food by sheep and on related problems. Br J Nutr 10, 69-91

Bouyssou T (1987) Variations d'origine alimentaire de la motricité digestive chez le lapin : nature et contrôle nerveux. Thèse de doctorat, Inst Nat Polytech de Toulouse, $210 p$

Bouyssou T, Candau M, Ruckebusch Y (1986) Sur l'intérêt de la mesure du transit digestif chez le lapin à l'aide de particules indigestibles. Ann Zootech 35, 401-410

Ellis WC, Matis $\mathrm{JH}$, Lascano $\mathrm{C}$ (1979) Quantitating ruminal turnover. Fed Proc 38, 2702-2706

Faichney GJ (1975) The use of markers to partition digestion within the gastro-intestinal tract of ruminants. In: Digestion and Metabolism in the Ruminant (IW McDonald, ACI Warner, eds) Univ New England Pub Unit, Armidale, NSW, Australia, 227241

Fioramonti J, Sorraing JM, Licois D, Bueno L (1981) Intestinal motor and transit disturbances associated with experimental coccidiosis (Eimeria magna) in the rabbit. Ann Rech Vet 12, 413-420

Furuichi Y, Takahashi T (1984) Movements of watersoluble and insoluble markers in the digestive tract of rabbits. Jpn J Zootech Sci 55, 552-561

Gidenne T (1985) Digestion chez le lapin en croissance, d'une ration à taux élevé de constituants pariétaux : étude méthodologique pour le calcul de digestibilité apparente par segment digestif. Ann Zootech 34 . $429-446$

Gidenne T (1987) Utilisation digestive de rations riches en lignines chez le lapin en croissance : mesures de flux et de transit dans différents segments digestifs. Ann Zootech 36, 95-108

Gidenne T (1993) Measurement of the rate of passage in restricted-fed rabbits: effect of dietary cell wall level on the transit of fibre particles of different sizes. Anim Feed Sci Technol 42, 151-163

Gidenne T, Bouyssou T, Ruckebusch Y (1988) Sampling of digestive contents by ileal canulation in the rabbit. Anim Prod 46, 147-151

Gidenne T, Carré B, Ségura M, Lapanouse A, Gomez J (1990) Fibre digestion and rate of passage in the rabbit: effect of particle size and level of lucerne meal. Anim Feed Sci Technol 32, 215-222

Grovum WL, Williams VJ (1973) Rate of passage of digesta in sheep. IV. Passage of marker through the alimentary tract and the biological relevance of rateconstants derived from the changes in concentration of marker in faeces. Br J Nutr 30, 313-329

Herrmann A (1989) Investigations about the influence of fibre and starch levels of diets on gastro-intestinal contents composition in young rabbits. Veterinar- medizinische Dissertation, Tierärztliche Hochschule, Hannover, $111 \mathrm{p}$

Jilge $B$ (1974) Soft faeces excretion and passage time in the laboratory rabbit. Lab Anim 8, 337-346

Jilge B (1980) Selective cannulation of small-sized marker particles during hard feces excretion. Proc 2 nd congress of the World Rabbit Science Association, Barcelone, Espagne, avril 1980 (WRSA ed), vol 1, 62-67

Jilge B (1982) Rate of movement of marker particles in the digestive tract of the rabbits. Lab Anim 16, 7-11

Jolivet E, Laplace JP, Lebas F (1975) Le transit digestif chez le lapin. IV. Essais de modélisation des transferts d'un marqueur radioactif entre les différents compartiments digestifs. Ann Zootech 24, 475-488

Lallès JP, Delval E, Poncet C (1991) Mean retention time of dietary residues within the gastrointestinal tract of the young ruminant: a comparison of noncompartmental (algebric) and compartmental (modelling) estimation methods. Anim Feed Sci Technol $35,139-159$

Laplace JP (1978) Le transit digestif chez les monogastriques. III. Comportement (prise de nourriture, cæcotrophie), motricité et transit digestif et pathogénie des diarrhées chez le lapin. Ann Zootech 27. 225-265

Laplace JP, Lebas F, Rioperez J (1975) Le transit digestif chez le lapin. II. Répartition de la radioactivité après ingestion d'aliment marqué au Cérium 41. Ann Zootech 24, 59-68

Lebas F, Laplace JP (1974) Note : sur l'excrétion fécale chez le lapin. Ann Zootech 23, 577-581

Leng E, Clauss W, Hornicke H (1977) Colon passage time in rabbits in relation to the formation of caecotrophes. Zbl Vet Med A 24, 324-332

Luick BR, Ayers A, Cheeke PR (1992) Rate of passage of Black Locust leaf and alfalfa meal in the rabbit gut. J Appl Rabbit Res 15, 914-921

Mambrini M (1990) Étude du temps de séjour des résidus alimentaires dans le tube digestif des vaches laitières : aspects méthodologiques et facteurs de variations. Thèse de Doctorat, univ Rennes I, 1990, $180 \mathrm{p}$

Moore JA, Pond KR, Poore MH, Goodwin TG (1992) Influence of model and marker on digesta kinetics estimates for sheep. J Anim Sci 70, 3528-3540

Morisse JP (1982) Le syndrome parésie cæcale-cedème pulmonaire chez le lapin. L'éleveur de lapins 17, 6971

Najar T, Sauvant D, Poncet C, Giger Reverdin S (1990) Comparaison de différents modèles d'ajustement des courbes d'excrétion fécale des marqueurs de transit. Repr Nutr Dévelop 30, suppl 1, 179-180

Nielsen CA (1991) Pathophysiology of infections by the gastric Trichostrongylid obeliscoides in a rabbit model system. Thesis Ph D, $197 \mathrm{p}$ 
Pickard WD, Stevens CE (1972) Digesta flow through the rabbit large intestine. Am J Physiol 222, 11611166

Piekarz R (1963) Effet de la coprophagie sur le temps de transit digestif chez le lapin domestique. Acta Physiol Polon 14, 359-370

Sakaguchi E, Kaizu K, Nakamichi M (1992) Fibre digestion and digesta retention time from different physi- cal forms of the feed in the rabbit. Comp Biochem Physiol 102A, 559-563

SAS (1988) SAS/STAT Guide for personal computers, version 6.03, Edition SAS (Statistical Analysis System) Institute Inc; Cary, NC, USA, 1028 p

Van Soest PJ, Robertson JB, Lewis BA (1991) Methods for dietary fiber, neutral detergent fiber, and nonstarch polysaccharides in relation to animal nutrition. J Dairy Sci 74, 3583-3597 